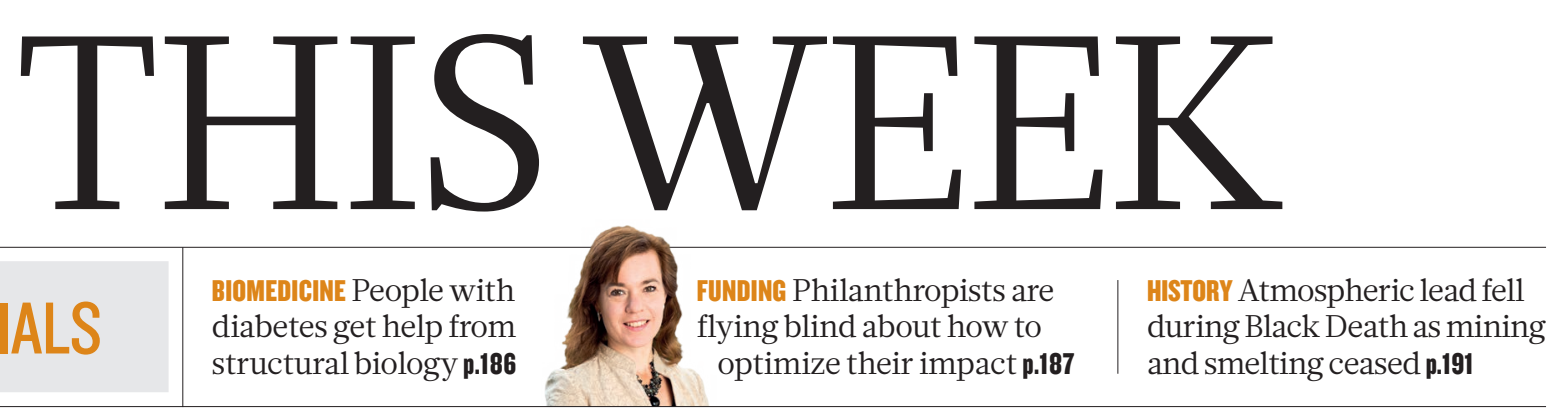

EDITORIALS

diabetes get help from structural biology p.186 flying blind about how to

optimize their impact $\mathbf{p . 1 8 7}$ during Black Death as mining and smelting ceased $\mathbf{p} .191$

\title{
On the pill
}

\section{A notable anniversary highlights the progress and benefits of contraceptives, and the continuing struggle for access to them.}

S ixty years ago, the US Food and Drug Administration approved a treatment for menstrual disturbance. Data showed that it prevented pregnancy too, but that use was taboo. Three years passed before doctors could prescribe it as a contraceptive. And another decade went by until many women could get it before marriage. By then, the drug was considered a miracle and was already famous enough to be known simply as the pill.

In 2015, the contraceptive-drug market was worth more than US $\$ 6.1$ billion globally, according to a report by Global Market Insights. And the pill has transformed society, or at least those societies in which most women have access to the drug. Epidemiologists have shown how contraceptives led to a rise in education and earnings for women in the United States and Western Europe. For example, a 2007 report analysed data from the 1960s and 1970s, and found that college enrolment in the United States was 17\% higher among women from states in which the pill was prescribed at 18 than among women from states that withheld permission until 21. And along with basic education, the pill has slowed population growth, a trend that can be linked to economic and environmental gains. In 1970, women in Bangladesh had an average of seven children; today they have two.

Large gaps in access remain, driven by sexism, poverty and cultural taboos. In South Africa, contraceptives have long been legal, but young women complain that nurses at reproductive-health-care clinics shame them for asking about sex. Three times more teenage girls have children in South Africa than in the United Kingdom (4.4\% compared with $1.4 \%)$. HIV rates in South Africa are the highest in the world, and the regions with high HIV tend to be those with high teen pregnancies.

Time and again, history shows how shame merely forces behaviour underground. HIV researchers know this, which is why labs running trials on treatments to prevent the disease offer reproductive health care as well. For this reason, they have voiced concerns over US President Donald Trump's 'gag rule', which reinstates a policy whereby US funds cannot go to any organization working abroad that discusses abortion (government funds have never been permitted for abortion itself). Salim Abdool Karim, director of the Durban-based South African AIDS research centre CAPRISA, points out that the centre now risks losing research funds from the US National Institutes of Health if a nurse at one of its satellite clinics merely addresses an HIV study participant's query on an unwanted pregnancy.

The Trump administration is poised to take direct aim at contraceptives in other ways. Proposed budget cuts to the Department of State and the United States Agency for International Development slash funds for services providing contraceptives for women in developing nations by $\$ 523$ million. A report from the Guttmacher Institute, a reproductivehealth think tank in Washington DC, estimates that, for every \$10-million drop in family-planning funds, 433,000 fewer women will receive contraceptives, which will result in 128,000 more unintended pregnancies. Studies have shown that a portion of these result in abortion,

which can be lethal to the women in places where abortion is illegal or inaccessible. A 2011 study posted on the Bulletin of the World Health Organization showed how the gag policy increased abortions when it was enacted in 2001 under president George W. Bush. The authors compared data between 1994 and 2008 from countries in sub-Saharan Africa that were receiving a high amount of US funding for reproductive health care with those receiving none. They found that abortion remained stable during this time in the latter countries, and rose

"In 1970, women in Bangladesh had an average of seven children; today they have two." sharply after 2001 in countries where funds had been cut.

Political battles and stigma have always accompanied the pill, yet the drug has stumbled towards progress over the decades. This year, a small company named Cadence moved forward with large studies to test whether women follow instructions on oral-contraceptive packs such that these might be sold over the counter. Both the American Medical Association and the American Congress of Obstetricians and Gynecologists support the measure.

A century ago, nurse Margaret Sanger fled the United States after being charged with obscenity for spreading information on birth control. In the Netherlands, she was inspired by a birth-control clinic and returned home to start her own. Later, she founded Planned Parenthood. She might be dismayed to know that millions of women still have no access to contraceptives because of a lack of political will. Or maybe she would be celebrating, because times for many other women have surely changed for the better.

\section{Line of beauty}

\section{The strange behaviour of $\mathrm{B}$ mesons may hold clues to what lies beyond the standard model.}

$\mathrm{T}$ The world's most powerful particle accelerator, the Large Hadron Collider (LHC), works incredibly well, but the next rung of understanding still seems to be just out of reach. Grasping it looks increasingly like a game of patience and precision, rather than just jumping higher. An effort to explore rare decays of 'beauty' particles is among the most promising lines of enquiry.

Researchers are desperate to unravel physics beyond the standard model, a description of particles and their interactions. Developed in the 1970s to unify three of the four fundamental forces, the standard model is concise enough to fit on a mug, and its predictions have been verified with dizzying precision. That culminated in the 2012 
discovery of the Higgs boson by the LHC at CERN, Europe's particlephysics laboratory near Geneva, Switzerland.

But physicists know that the standard model is not the end of the story, not least because it doesn't include gravity or explain dark matter. It is at best part of a wider undiscovered framework, or an approximation of a more fundamental theory. At the optimistic start-up of the LHC in 2009, the path beyond the standard model seemed marked out. Many theorists assumed that particles predicted by an elegant extension to the model - supersymmetry - would reveal themselves as soon as colliders could reach a high enough energy.

Since then, LHC experiments have smashed together quadrillions of particles at record energies, and no supersymmetric particles have emerged. Proponents have not given up hope, but they have ruled out the simplest versions of their theories. Now, rather than rely on conjuring new particles, many are hoping to observe their effects indirectly, prying open tiny cracks in the standard model by exploring a handful of findings that don't quite fit predictions. Two reviews in this week's Nature examine one area in which anomalies are accruing (see pages 221 and 227). Excitement is growing about the strange and subtle behaviour of particles called $B$ mesons, whose properties make them ripe for observing the effects of new physics.

CERN's LHCb and other ' $B$-factory' experiments, such as Belle at Japan's High Energy Accelerator Research Organization (KEK) in Tsukuba, have often worked in the shadows of their more famous sister experiments. Eschewing the need for higher and higher energies, $B$ factories hoover up data on rare decays of $B$ mesons, which contain $b$ quarks (denoting 'beauty' or 'bottom'). Physicists make precise measurements of how $B$ mesons decay into known particles, searching for tiny discrepancies that would reveal the influence of hypothetical heavy particles that appear fleetingly but leave their mark.

Several such discrepancies exist in various $B$-meson decays, any of which on its own would not raise an eyebrow. But together they seem to fit into a broader picture. Each anomaly hints at the existence of the same

exotic new particle. Its identity is not yet known, but candidates include a heavier type of $Z$ boson - a $Z$ ' - or a 'leptoquark'. Some see this as a wishful cobbling together of statistical flukes: most of the discrepancies are just a few standard deviations away from expectations, and far from the five standard deviations that physicists require to declare a discovery. Yet the strongest of the anomalies - in the rates at which $B$ mesons turn into pairs of different kinds of lepton (electrons and their heavier cous-

"Rather than rely on conjuring new particles, many hope to pry open cracks in the standard model." ins, muons and taus) - has been seen by three separate experiments, whose combined data give a tantalizing standard deviation of four.

Physicists have found few other anomalies to lead them beyond standard-model physics. One involves looking at a deviation in how muons wobble on their axes as they travel in a magnetic field. Many experiments are probing neutrinos - ghostly particles that the standard model assumed were massless, but turned out not to be, and whose basic behaviour physicists are just starting to understand. Meanwhile, other once-promising leads are fading. The proton-radius puzzle - in which experimental physicists disagree over the ubiquitous particle's size by as much as $4 \%$ - could well be down to an incomplete understanding of the measurement process. And a shortage in the number of antineutrinos arriving at experiments near nuclear reactors looks increasingly likely to be caused by errors in calculating the predicted rate.

Beauty physics offers a rare glimmer of hope. The LHCb will continue to rack up data, and KEK's upgraded $B$-factory detector, Belle II, starts up next year. If anomalies fade with more data, a planned upgrade to the LHC - which will ramp up the intensity of collisions from 2025 - could provide a new proving ground.

Discovery in high-energy physics is turning out to be less like swashbuckling through Terra Incognita than some might have hoped. But groping for tiny cracks can also reveal unknown territory - it just takes more patience.

\section{Rescue remedy}

\section{Life-saving treatments for diabetes could come from new work on solving receptor structures.}

$\mathrm{M}$ ost people know about mouth-to-mouth resuscitation and chest compressions, even if they have never had to put them into practice. Some could probably attempt the Heimlich manoeuvre. But how many have heard of glucagon rescue?

To a person with diabetes who has dangerously low blood sugar, a shot of the hormone glucagon delivered by someone else is their best chance of survival. Many carry a life-saving glucagon rescue kit. But it's difficult to use in a hurry. A powder must be mixed into solution, shaken and then delivered with a hypodermic syringe. Even people who are practised struggle. To those who are unfamiliar, the lengthy instructions can be enough to stop them from trying. If a pupil falls into a hypoglycaemic coma, teachers in some US schools have it written into their contracts that they are to call the emergency services instead.

So research that could lead to a better way to address hypoglycaemia and other complications of diabetes is an urgent pursuit. This week, Nature publishes four papers that describe such work. At first glance, the research might seem far removed from the medical front line. It's hardcore structural biology: solved crystal and cryo-electron microscopy (cryo-EM) structures of G-protein-coupled receptors. But the insights could improve glucagon rescue, and lead to other treatments for diabetes and obesity.

In the body, glucagon triggers the conversion of stored glycogen to glucose - the opposite effect to insulin. Stable synthetic insulin is routine in medicine, but glucagon poses more of a problem. Solutions of it go off, hence the tricky rescue kits. Research that could make glucagon solutions stable and rescue kits simpler would have a life-saving impact. How to achieve that? The hormone increases glucose levels by triggering a biochemical sequence that is heavily dependent on a signalling protein. A related signalling protein activates insulin to mop up excess glucose. Type 2 diabetes causes problems because it disrupts this balance. The full structure of these signalling proteins was unknown - until now.

The two proteins are glucagon-like peptide-1 receptor (GLP-1R) and glucagon receptor (GCGR). Their activation and deactivation have opposing roles in glucose homeostasis and insulin release, and so help to regulate metabolism and appetite. They are activated by peptides, which could be adapted as potential drugs. Design of peptide or small-molecule drugs requires data on how and where the molecules bind - information now revealed in the structures. Indeed, in one of the studies, researchers designed a new peptide molecule that activates GLP-1R in mice (A. Jazayeri et al. Nature 546, 254-258; 2017).

Another describes the use of cryo-EM to solve the GLP-1R activated structure in complex with its $\mathrm{G}$ protein signalling partner (Y. Zhang et al. Nature 546, 248-253;2017). It shows how the receptor grabs and secures the peptide, then twists to pass on the signal. Finally, two others report the structures of GLP-1R and GCGR in their inactive forms - both deactivated by small molecules that bind to a different site from the natural hormone (G. Song et al. Nature 546, 312-315 (2017) and H. Zhang et al. Nature 546, 259-264 (2017)). Because small-molecule drugs are easier to design than peptide-hormone mimics, these structures suggest new therapeutic opportunities for regulating glucose homeostasis.

The need is great: in trials, even parents trained to use the kits on their children took an average of 2.5 minutes to mix and deliver glucagon. And many injected nothing but fresh air. They need a helping hand. Structural biology is trying to provide just that. 